\title{
EVALUACIÓN DE INSECTICIDAS NO CONVENCIONALES \\ PARA EL CONTROL DE Aphis gossypii Glover \\ (HEMIPTERA: APHIDIDAE) EN FRÍJOL
}

\section{EVALUATION OF NON-CONVENTIONAL INSECTICIDES FOR THE CONTROL OF Aphis gossypii Glover (HEMIPTERA: APHIDIDAE) IN BEANS}

\author{
Manuel J. Peña ${ }^{1}$, Julio C. Castro ${ }^{2}$, Alberto Soto ${ }^{3}$ \\ ${ }^{1}$ Especialista en Agroecología Tropical Andina. Universidad Santa Rosa de Cabal, Colombia; manueljp75@misena.edu.co ${ }^{2}$ \\ Especialista en Agroecología Tropical Andina. Universidad Santa Rosa de Cabal, Colombia; jucecavi@misena.edu.co ${ }^{3}$ I.A., \\ M.Sc., Ph.D. Profesor Titular Universidad de Caldas. Calle 65 No 26-10 Manizales, Caldas, Colombia; alberto.soto@ucaldas. \\ edu.co
}

Rev. U.D.C.A Act. \& Div. Cient. 16(1): 131 - 138, 2013

\section{RESUMEN}

La concentración letal (CL) es usada para estimar la toxicidad de pesticidas a artrópodos; sin embargo, la CL es una medida incompleta de los efectos de los productos sobre poblaciones, pues analiza solamente la mortalidad, como parámetro de toxicidad. Se sabe que individuos que sobreviven a la exposición a pesticidas pueden sufrir efectos subletales. En este trabajo fue estudiado en laboratorio los efectos letales y subletales del caldo sulfocálcico, Azadirachta indica (Bioneem ${ }^{\circledR}$ ) y Allium sativum-Capsicum chinense (Alisin ${ }^{\circledR}$ ) en el crecimiento poblacional de A. gossypii y el efecto fitotóxico de estos insecticidas sobre plantas de fríjol. Los efectos letales fueron estimados a través de test de toxicidad aguda con obtención de curvas dosis-respuesta y sus respectivas concentraciones letales y los efectos subletales, a través de la estimativa de la tasa instantánea de crecimiento poblacional $\left(\mathrm{r}_{\mathrm{i}}\right)$. Las concentraciones letales $\left(\mathrm{CL}_{95}\right)$ y subletales $\left(\mathrm{CL}_{50}\right)$ del Caldo sulfocálcico fueron 0,64 y 0,25\% del producto, respectivamente, para el Bioneem ${ }^{\circledR}$, de 1,22 y $0,71 \%$ del producto, y para el Alisin ${ }^{\circledR}$, de 2,18 y $0,94 \%$ del producto, lo que indica que se podrían utilizar para el control del fitófago. No se presentaron síntomas graves de fitotoxicidad sobre las plantas asperjadas. El manejo de A. gossypii en fríjol es posible de ser realizado con los productos Caldo sulfocálcico, Bioneem ${ }^{\circledR}$ y Alisin ${ }^{\circledR}$.

Palabras clave: Áfidos, Azadirachta indica, azufre, productos alternativos.

\section{SUMMARY}

The lethal concentration (CL) is used to estimate the toxicity of pesticides in arthropods; however, CL is an incomplete measure of the effects of these products on populations, since it only examines toxicity as a function of mortality. It is known that individuals which survive the exposure to pesticides may suffer sublethal effects. This work studied, under laboratory conditions the lethal and sublethal effects of lime sulphur, Azadirachta indica (Bioneem ${ }^{\circledR}$ ) and Allium sativumCapsicum chinense (Alisin $\AA$ ) on the population growth of A. gossypii and the phytotoxic effect of these insecticides on bean plants. Lethal effects were estimated through tests of acute toxicity to obtain dosage-response curves and their respective lethal concentrations and sublethal effects by estimating the instantaneous population growth rate $\left(\mathrm{r}_{\mathrm{i}}\right)$. The lethal and sublethal concentrations of lime sulfur against were 1.22 and $0.25 \%$ of the product, respectively, for the Bioneem ${ }^{\circledR}$ were 1.22 and $0.71 \%$ of the product, respectively, and the Alisin ${ }^{\circledR} 2.18$ and $0,94 \%$ of the product, indicating that it could be used to control this phytophagous insect. There were no severe symptoms of phytotoxicity detected on sprayed plants. The management of $A$. gossypii in beans can be made with lime sulphur, Bioneem ${ }^{\circledR}$ and Alisin ${ }^{\circledR}$.

Key words: Aphids, Azadirachta indica, sulfur, alternative products. 


\section{INTRODUCCIÓN}

Aphis gossypii Glover, 1877 (Hemiptera: Stenorrhyncha: Aphididae) es una plaga cosmopolita y altamente polífaga, presente en más de 90 familias de plantas (Michelotto et al. 2004). Las altas poblaciones de este insecto ocasionan deformación y encrespamiento de las hojas y retraso en el crecimiento de las plantas, además de ser importantes agentes transmisores de virus (Bueno, 2005).

El control químico sigue siendo la herramienta más utilizada por los productores para el control de la plaga; sin embargo, a pesar de su eficiencia, presenta aspectos negativos, como selección de individuos resistentes, debido al uso continuo de ingredientes activos, reducción o supresión de especies benéficas, alta toxicidad de los productos a los aplicadores, presencia de residuos en los alimentos y, en el medio ambiente, fitotoxicidad, entre otros (Filgueira, 2000; Easterbrook et al. 2001; Fragoso et al. 2002; Picanço et al. 2007).

Una alternativa viable a los problemas ocasionados por el uso excesivo de plaguicidas sintéticos en el cultivo de fríjol es la utilización de nuevos ingredientes activos que deben priorizar la seguridad ambiental y social y que sean eficientes en el control de A. gossypii. En la búsqueda de tales sustancias, productos alternativos, como los caldos fitoprotectores y los extractos de plantas, han sido usados por productores de hortalizas para control de plagas y enfermedades, especialmente, en los sistemas de producción ecológicos y orgánicos (Penteado, 2000; Campanhola \& Bettiol, 2003; Venzon et al. 2007; Soto et al. 2011).

Dentro de esos productos, se encuentra el nim Azadirachta indica A. Juss, cuyo principal compuesto activo es la azadirachtina, un tetranortriterpenoide aislado de las semillas (Rembold, 1989; Martínez, 2002), que presenta elevada acción insecticida y acaricida, bajísima toxicidad al hombre y animales domésticos, selectividad a los enemigos naturales, además de no agredir el ambiente (Mansour \& Ascher, 1983; Spollen \& Isman, 1996; Momen et al. 1997; Castagnoli et al. 2000; El-Gengaihi et al. 2000; Mourão et al. 2004; Brito et al. 2006; Soto et al. 2011).

El ajo, Allium sativum y el ají, Capsicum, se han utilizado para el control de insectos minadores, chupadores, barrenadores y masticadores. Estas plantas presentan acción repelente y actúan por ingestión, causando trastornos digestivos, por lo tanto, el insecto deja de alimentarse. El Alisin ${ }^{\circledR}$ es un producto comercial a base de ají-ajo utilizado por los agricultores, para el control de plagas en hortalizas (Vásquez, 2005).

El caldo sulfocálcico es otro producto alternativo empleado para el control de plagas, obtenido por el tratamiento térmi- co del azufre y de la cal. El efecto tóxico de ese producto a los insectos y ácaros es debido a la liberación de gas sulfídrico $\left(\mathrm{H}_{2} \mathrm{~S}\right)$ y azufre coloidal, cuando se aplica sobre las plantas (Abbot, 1945). El uso del Caldo sulfocálcico ha sido relatado con éxito para el control de insectos y de ácaros (Wardlow \& Ludlam, 1975; Guerra, 1985; Penteado, 2000; Guirado, 2001; Ocete et al. 2003; Venzon et al. 2007; Soto, 2009; Soto et al. 2011).

En este trabajo, se evaluó el potencial del caldo sulfocálcico $\left(31,5^{\circ} \mathrm{B}\right)$ y de formulaciones comerciales a base de nim Bioneem ${ }^{\circledR}$ y de Ají-ajo Alisin ${ }^{\circledR}$, para el control de A. gossypii. Inicialmente, se avaluó la toxicidad letal, a través de la estimativa de la concentración letal (CL) de los productos; sin embargo, como la CL es una medida incompleta de los efectos de los productos sobre poblaciones, pues analiza solamente la mortalidad como parámetro de toxicidad (Stark \& Banks, 2003); se apreció también los efectos subletales de los productos, por medio de la estimativa de la tasa instantánea de crecimiento poblacional (ri) (Stark \& Rangus, 1994). Posteriormente, fue evaluado en invernadero la fitotoxicidad que causan dichos productos sobre las plantas de fríjol asperjadas.

\section{MATERIALES Y MÉTODOS}

El trabajo, se realizó en el laboratorio de Entomología del Departamento de Producción Agropecuaria, de la Universidad de Caldas, Manizales. La cría de A. gossypii fue iniciada con áfidos colectados en plantas de fríjol provenientes de la granja Tesorito de la Universidad de Caldas y mantenida en plantas de fríjol infestadas con el insecto y ubicadas en jaulas de madera, forradas con muselina. Fueron estudiados los efectos letales y subletales del caldo sulfocálcico a $31,5^{\circ} \mathrm{Bau}-$ mé y de los productos comerciales Bioneem ${ }^{\circledR}(3,3 \mathrm{mg} / \mathrm{L}$ de Azadirachtina) y Alisin ${ }^{\circledR}(70 \mathrm{~g} / \mathrm{L}$ de ají-ajo) sobre A. gossypii. Los efectos letales fueron estimados a través de test de toxicidad aguda con obtención de curvas dosis-respuesta y sus respectivas concentraciones letales (CL) y los efectos subletales, por medio de la estimativa de la tasa instantánea de crecimiento poblacional $\left(\mathrm{r}_{\mathrm{i}}\right)$.

Fueron estimadas las concentraciones letales del Caldo sulfocálcico, que fue preparado de acuerdo con metodología adaptada de Penteado (2000), utilizando tratamiento térmico del azufre y cal virgen (para cada litro de agua, $250 \mathrm{~g}$ de azufre y $125 \mathrm{~g}$ de cal virgen) y de los productos Bioneem ${ }^{\circledR}$ y Alisin ${ }^{\circledR}$, para hembras adultas de A. gossypii. Las concentraciones evaluadas fueron escogidas a través de bioensayos iniciales y se situaron entre el límite inferior, donde el producto no causó mortalidad y el límite superior de respuesta, donde generó $100 \%$ de mortalidad.

Los bioensayos de concentración-respuesta fueron realiza- 
dos utilizándose hembras del áfido A. gossypii al inicio de la fase reproductiva. Los productos fueron pulverizados en discos $(\varnothing=3,0 \mathrm{~cm})$ de hojas de fríjol, acondicionados en caja de Petri, por medio de aspersor manual Brudden ${ }^{\circledR}$ modelo S.S., con capacidad de cinco litros, provisto con una boquilla tipo cono regulable, con una presión de $5 \mathrm{lb} / \mathrm{pul}^{2}$ y con aplicación de un volumen igual a $2,5 \mathrm{~mL}$, lo que corresponde a un depósito de $1,70 \pm 0,07 \mathrm{mg} / \mathrm{cm}^{2}$ sobre la superficie tratada

Los discos asperjados con los productos y con agua (testigo) fueron expuestos al ambiente por una hora. Posteriormente, 10 hembras de $A$. gossypii fueron colocadas sobre cada disco; cada concentración del producto fue repetida cinco veces. Los discos de hojas tratados fueron mantenidos en cámara climatizada $\left(25 \pm 2^{\circ} \mathrm{C}, 60 \pm 10 \% \mathrm{HR}\right.$ y $12 \mathrm{~h}$ de luz); la mortalidad fue evaluada 24 h después a la aplicación de los productos y las curvas de concentración-mortalidad fueron estimadas por el análisis de Probit (Finney, 1971).

La evaluación de la acción subletal de los productos sobre el áfido A. gossypii fue realizada utilizándose la misma metodología citada para la evaluación de la acción letal de los productos; el tiempo de evaluación fue de 15 días.

Para cada concentración de cada producto fueron ejecutadas cinco repeticiones, donde cada una fue representada por un disco con diez hembras de A. gossypii. Los áfidos fueron mantenidos en cámaras climatizadas sobre las mismas condiciones descritas en los experimentos de toxicidad letal. La evaluación del efecto subletal, se realizó a través de la estimativa de la tasa instantánea de crecimiento $\left(r_{i}\right)$, por la siguiente fórmula (Stark \& Banks, 2003):

$$
\mathrm{r}_{\mathrm{i}}=\ln \left(\mathrm{N}_{\mathrm{f}} / \mathrm{N}_{0}\right) / \Delta \mathrm{t}
$$

Donde $\mathrm{N}_{\mathrm{f}}$ es el número final de individuos; $\mathrm{N}_{0}$ es el número inicial de individuos y $\Delta \mathrm{t}$ es la variación de tiempo (duración del experimento 15 días). El valor positivo de $r_{i}$ significa que la población está en crecimiento; $\mathrm{r}_{\mathrm{i}}=0$, indica que la población está estable, mientras que un valor negativo de $\mathrm{r}_{\mathrm{i}}$ señala que la población está en descenso y en vías de extinción. El análisis de regresión, se aprovechó para la evaluación de $\mathrm{r}_{\mathrm{i}}$ en función de las concentraciones utilizadas.

Fueron evaluados los síntomas visuales de fitotoxicidad en plantas de fríjol variedad calima con 30 días de edad, para cada tratamiento (caldo sulfocálcico 0,25 y 0,64\%, Bioneem ${ }^{\circledR} 0,71$ y $1,22 \%$, y Alisin ${ }^{\circledR} 0,94$ y $2,18 \%$ ), por dos observadores, a través de la siguiente escala visual de notas: Nota 0: Plantas con hojas normales, sin síntoma de quemaduras; Nota 1: Plantas con hojas levemente lesionadas o con pequeñas áreas quemadas; Nota 2: Plantas con hojas medianamente quemadas, amarillas y con bordes y ápices quemados; Nota 3: Plantas con hojas intensamente lesionadas, presentando severa defoliación.

Los datos de fitotoxicidad fueron transformados para $\operatorname{arcsen} \sqrt{x / 100}$ y sometidos a análisis de varianza del Modelo Lineal General. Este tipo de análisis fue hecho usando el indicador Wilks' Lambda (SAS Institute, 1989), versión 9.0 con especificación PROFILE.

\section{RESULTADOS Y DISCUSIÓN}

Toxicidad letal: Para el Caldo sulfocálcico fueron obtenidas $\mathrm{Cl}_{50}$ de 0,25 y la $\mathrm{Cl}_{95}$ de $0,64 \%$ de concentración del producto a $31,5^{\circ}$ B. Para el Bioneem ${ }^{\circledR}$ fueron obtenidas $\mathrm{Cl}_{50}$ de 0,71 y la $\mathrm{Cl}_{95}$ de $1,22 \%$ de ese producto. Para el Alisin ${ }^{\circledR}$ fueron obtenidas $\mathrm{Cl}_{50}$ de 0,94 y la $\mathrm{Cl}_{95}$ de $2,18 \%$ de concentración del producto (Tabla 1).

Tabla 1. Toxicidad de los productos alternativos al áfido A. gossypii.

\begin{tabular}{|c|c|c|c|c|c|c|}
\hline Producto & $\mathbf{N}^{1}$ & $\begin{array}{c}\text { Coeficiente } \\
\text { Angular } \pm \mathrm{EP}^{2}\end{array}$ & $\begin{array}{c}\mathrm{Cl}_{50}{ }^{3} \\
\text { (I.C. } \text { a 95\%) }\end{array}$ & $\begin{array}{c}\mathrm{Cl}_{95}{ }^{4} \\
\text { (I.C. } \text { a 95\%) }\end{array}$ & $\mathbf{X}^{2}$ & $\mathbf{P}$ \\
\hline Caldo sulfoc. & 150 & $0,21 \pm 0,05$ & $0,25(0,20-0,32)$ & $0,64(0,38-0,70)$ & 0,63 & 0,41 \\
\hline Bioneem ${ }^{\circledR}$ & 220 & $0,33 \pm 0,12$ & $0,71(0,67-0,74)$ & $1,22(1,02-1,43)$ & 0,39 & 0,78 \\
\hline Alisin ${ }^{2}$ & 310 & $0,41 \pm 0,07$ & $0,94(0,81-1,32)$ & $2,18(2,01-2,44)$ & 0,92 & 0,64 \\
\hline
\end{tabular}

${ }^{1}$ Número de áfidos evaluados

${ }^{2}$ Coeficiente angular y error padrón de la media

${ }^{3}$ Concentración letal media e intervalo de confianza a 95\%

${ }^{4}$ Concentración letal que causa 95\% de mortalidad e intervalo de confianza a 95\% 
Tasa instantánea de crecimiento poblacional: La tasa instantánea de crecimiento poblacional de A. gossypii fue igual a cero, indicando que la población está estable en las concentraciones de 0,39\% del Caldo sulfocálcico; 0,56\% del producto Bioneem ${ }^{\circledR}$ y $1.43 \%$ del producto Alisin ${ }^{\circledR}$ (Figuras 1 a 3).

Evaluación de la fitotoxicidad: Con relación a la fitotoxicidad, el análisis de varianza con medidas repetidas en el tiempo indicó efectos significativos del producto $\left(\mathrm{F}_{2,9}=31,29 ; \mathrm{P}=\right.$ $<0,0001)$, de la concentración $\left(\mathrm{F}_{1,9}=6,32 ; \mathrm{P}=0,01\right)$ y de la interacción producto $\mathrm{x}$ concentración $\left(\mathrm{F}_{2,9}=3,01 ; \mathrm{P}=0,03\right)$ (Tabla 2). No se presentó efecto significativo entre el tiempo (Wilks' Lambda= 0,6539; F=0,51; GLnum= 2; GLden= $12 ; \mathrm{P}=0,56$ ) y de las interacciones producto $\mathrm{x}$ tiempo (Wilks' Lambda $=0$,3556; $F=1,58 ;$ GLnum $=6$; GLden = 31,25; $\mathrm{P}=0,14$ ), concentración $\mathrm{x}$ tiempo (Wilks' Lambda $=0,7928$; $\mathrm{F}=0,23$; GLnum $=2$; GLden $=12 ; \mathrm{P}=0,86)$ y producto $\mathrm{x}$

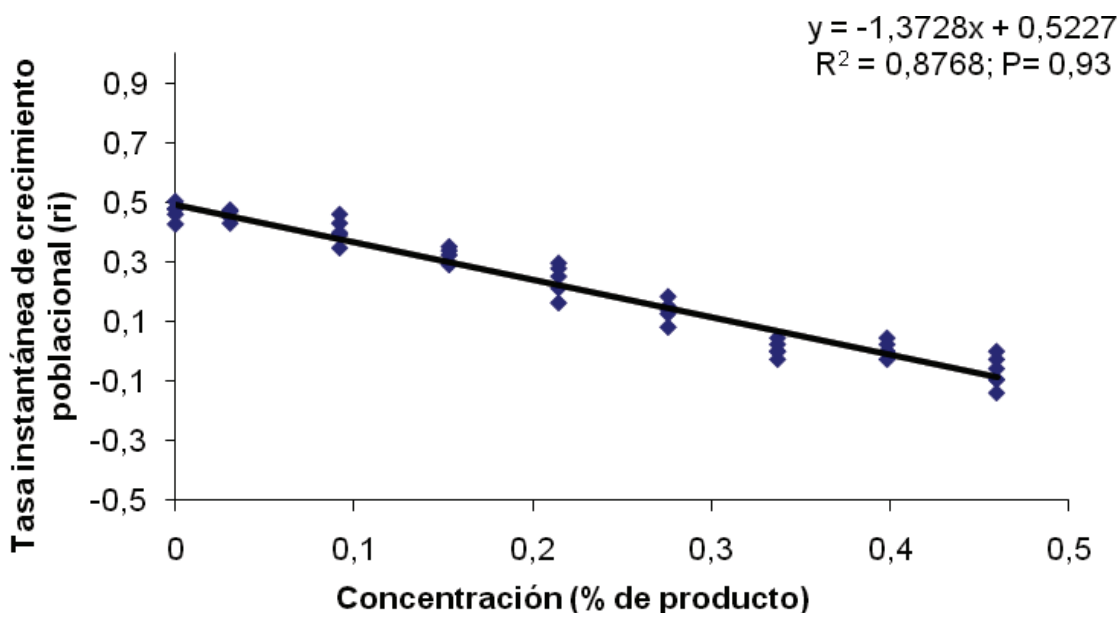

Figura 1. Tasa instantánea de crecimiento poblacional $\left(\mathrm{r}_{\mathrm{i}}\right)$ de A. gossypii en función de la aplicación de concentraciones del Caldo sulfocálcico.

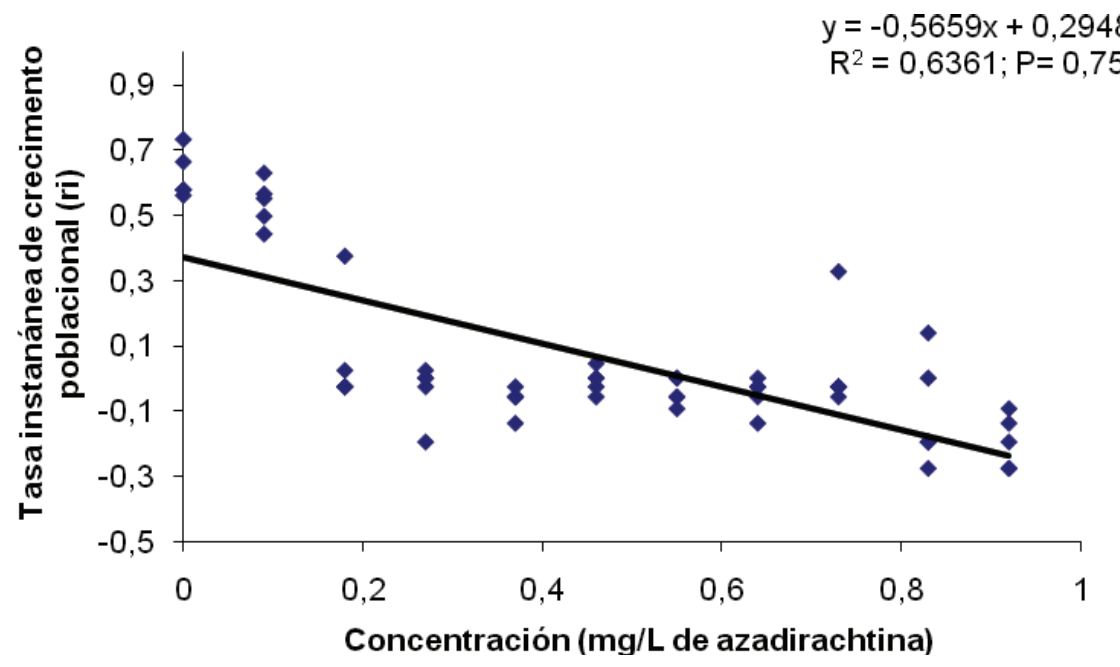

Figura 2. Tasa instantánea de crecimiento poblacional $\left(r_{i}\right)$ de A. gossypii en función de la aplicación de concentraciones de Bioneem ${ }^{\circledR}$. 


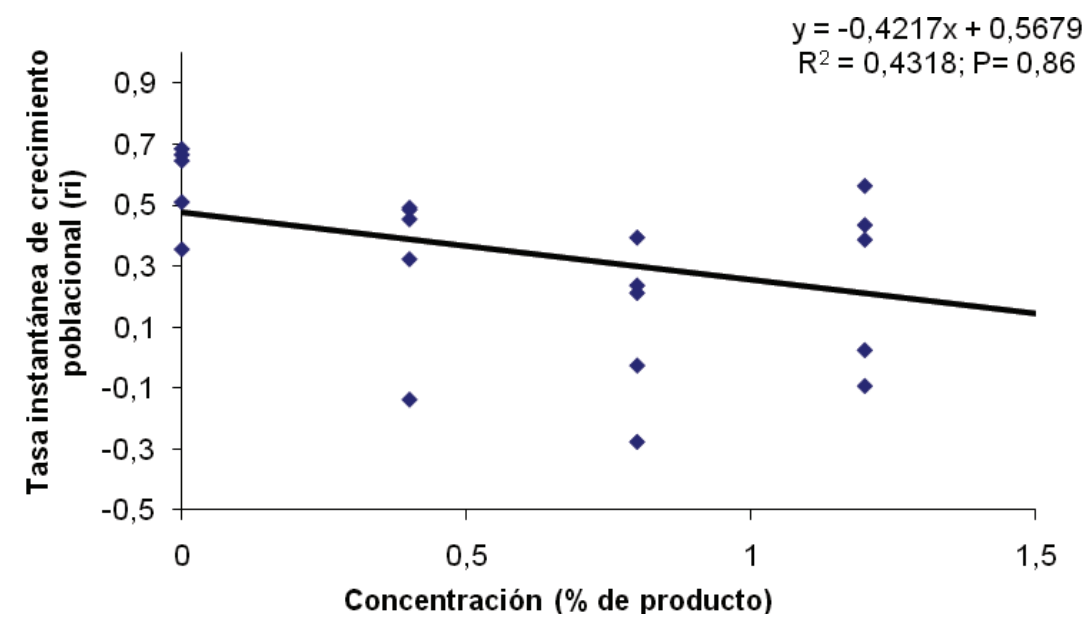

Figura 3. Tasa instantánea de crecimiento poblacional $\left(\mathrm{r}_{\mathrm{i}}\right)$ de A. gossypii en función de la aplicación de concentraciones del producto comercial Alisin ${ }^{\circledR}$.

concentración $\mathrm{x}$ tiempo (Wilks' Lambda $=0,5231 ; \mathrm{F}=0,89$; GLnum= 6; GLden= 31,25; P= 0,53) (Tabla 3). Solamente se observó baja fitotoxicidad en las plantas asperjadas con Caldo sulfocálcico hasta el quinto día después de la aplicación; los otros tratamientos no presentaron síntomas de fitotoxicidad.

Para que se obtenga un $95 \%$ de mortalidad de A. gossypii, las concentraciones del caldo sulfocálcico, Bioneem ${ }^{\circledR} \mathrm{y}$ Alisin ${ }^{\circledR}$ no fueron superiores a $0,64 \%, 1,22 \%$ y $2,18 \%$, respectivamente. Eso demuestra que estos productos muestran potencial para ser aplicados sobre el cultivo de fríjol, con el objetivo de controlar poblaciones de A. gossypii, actualmente considerado como una importante plaga que ataca varios cultivos (Soglia et al. 2002; Pallini et al. 2004). Con relación a las concentraciones del Caldo sulfocálcico requeridas para el control de A. gossypii, las concentraciones letales y subletales fueron menores que las recomendadas y utilizadas en sistemas de cultivo orgánico. Estas concentraciones varían de 2 a $4 \%$ del producto que posee una densidad de 29 a $32^{\circ}$ Baumé (Penteado, 2000; D’Andrea, 2001), aproximadamente, 0,58 a $1,28 \%$ de polisulfuro de calcio en el producto pulverizado. Breda et al. (2011) evaluaron el insecticida a base de Azadirachtina Azamax® y el extracto acuoso de semillas de nim en concentraciones de 0 a $2 \%$ y de 0 a 2,5\%, respectivamente, sobre el áfido $A$. gossypii, encontrando una mortalidad del 64 a $100 \%$ para Azamax® y de 12 a 92\%, para el extracto acuoso de nim.

Soto et al. (2010) evaluaron sobre el cultivo de fresa los productos Neem Pro ${ }^{\circledR}$, Organic Neem ${ }^{\circledR}$ y Natuneem ${ }^{\circledR}$ para el control de T. urticae (Acari: Tetranychidae), los cuales, controlaron el 95,28\% de la población siete días después de la aplicación de los mismos, sin afectar a su predador Phytoseiulus macropilis (Acari: Phytoseiidae). En otro trabajo, se evaluaron dichos productos para el control del ácaro Tetranychus evansi (Acari: Tetranychidae) en el cultivo de tomate, ocasionando el $82 \%$ de control de la población de la plaga, un día después de la aplicación (Soto et al. 2011).

Los productos caldo sulfocálcico, Bioneem ${ }^{\circledR}$ y Alisin ${ }^{\circledR}$ representan una alternativa para el control de plagas, especialmente, cuando los agrotóxicos no son permitidos, como en cultivos orgánicos. Otro punto importante a ser considerado

Tabla 2. Análisis de varianza entre productos y dosis de la fitotoxicidad de los productos alternativos.

\begin{tabular}{|l|c|c|c|}
\hline \multicolumn{1}{|c|}{ FV } & GL & F & P \\
\hline Producto & 2 & 31,29 & $<0,0001 *$ \\
\hline Concentración & 1 & 6,32 & $0,01 *$ \\
\hline Producto x concentración & 2 & 3,01 & $0,03 *$ \\
\hline Error & 12 & & \\
\hline
\end{tabular}


Tabla 3. Análisis de varianza entre tiempo, productos y dosis de la fitotoxicidad de los productos alternativos.

\begin{tabular}{|l|c|c|c|c|c|}
\hline \multicolumn{1}{|c|}{ FV } & Wilks' Lambda & F & $\begin{array}{c}\text { GL } \\
\text { numerador }\end{array}$ & $\begin{array}{c}\text { GL } \\
\text { denominador }\end{array}$ & P \\
\hline Tiempo & 0,6539 & 0,51 & 2 & 12 & 0,56 \\
\hline Producto x tiempo & 0,3556 & 1,58 & 6 & 31,25 & 0,14 \\
\hline Concent. $x$ tiempo & 0,7928 & 0,23 & 2 & 12 & 0,86 \\
\hline Producto x concent. $x$ tiempo & 0,5231 & 0,89 & 6 & 31,25 & 0,53 \\
\hline
\end{tabular}

es que para aplicaciones cuyos áfidos no presentan mortalidad inmediata, debido a la concentración aplicada, fueron estudiados los efectos retardados de estos productos, esto es, efectos subletales, que representan cuál es la concentración que lleva a la parálisis del crecimiento poblacional. El crecimiento poblacional de $A$. gossypii fue interrumpido cuando se usó el Caldo sulfocálcico, el Bioneem ${ }^{\circledR}$ y el Alisin ${ }^{\circledR}$ en concentración de 0,39\%, 0,56\%, 1,43\%, respectivamente. La obtención de control de A. gossypii a bajas concentraciones representa un gran avance en relación a los estudios, que buscan el control de ese áfido; sin embargo, el hecho que $A$. gossypii presente alta susceptibilidad, no implica que estos productos puedan ser aplicados indiscriminadamente en concentraciones más altas que las recomendadas, como resultado de experimentos científicos en campo o en invernadero, ya que se podría presentar problemas de fitotoxicidad.

Los efectos de las dosis subletales de los productos en la población se ven manifestados a través de la reducción en el periodo de vida, disminución de fertilidad, reducción de la fecundidad, cambios en el comportamiento sexual y en la alimentación (Stark et al. 1992); esto demuestra la importancia de utilizar concentraciones subletales de los productos alternativos para el manejo de A. gossypii. La decisión de usar concentraciones letales o subletales de los productos alternativos depende de la población plaga al momento de la aplicación. Los productos a base de nim y caldo sulfocálcico, en dosis subletales, presentan, normalmente, selectividad para los enemigos naturales (Dimetry et al. 1993; Castiglioni et al. 2002; Soto et al. 2011).

Estos resultados demuestran la eficiencia de la aplicación de los productos alternativos para el control de poblaciones de A. gossypii; sin embargo, la eficiencia de los productos alternativos para el control de plagas, así como la selectividad a enemigos naturales está relacionada con la dosis y con la formulación empleada. Es necesario tener un conocimiento técnico sobre el producto que se va a utilizar, para que se obtenga un control satisfactorio de las poblaciones de plagas, de manera que no afecte a los enemigos naturales asociados a estas (Soto et al. 2010).
La necesidad de encontrar productos alternativos a los agrotóxicos convencionales con potencial preventivo y curativo contra las plagas y que no presenten efectos dañinos al medio ambiente, consumidor y productor viene creciendo en todo el mundo. Por presentar esas características, los productos caldo sulfocálcico, Alisin ${ }^{\circledR}$ y Bioneem ${ }^{\circledR}$ pueden ser considerados como importantes alternativas para uso en la agricultura tradicional y orgánica. En general, los efectos benéficos de los productos alternativos muchas veces son colocados en duda, debido a la posible fitotoxicidad que los mismos pudieran provocar; no obstante, en este trabajo fueron evaluados los posibles efectos fitotóxicos ocasionados por la aplicación de los productos alternativos, encontrándose muy baja fitotoxicidad.

La aplicación de los productos Alisin ${ }^{\circledR}(0,94$ y $2,18 \%)$, Bioneem ${ }^{\circledR}(0,71$ y $1,22 \%)$ y el Caldo sulfocálcico $(0,25$ y $0,64 \%)$, representan una alternativa viable para el control de $A$. gossypii sobre plantas de fríjol, tanto cuando se aplican en las dosis letales como en las dosis subletales. En la utilización de dosis subletales es importante considerar el tiempo para la acción insecticida de esos productos.

Agradecimientos: Al Servicio Nacional de Aprendizaje SENA, Colombia, por el financiamiento de la investigación. Conflictos de intereses: El artículo fue preparado y revisado con la participación de todos los autores, quienes declaramos que no existe ningún conflicto de intereses que ponga en riesgo la validez de los resultados presentados.

\section{BIBLIOGRAFÍA}

1. ABBOT, C.E. 1945. The toxic gases of lime-sulfur. J. Econ. Entomol. 38(5):618-620.

2. BREDA, M.O.; OLIVEIRA, J.; MARQUES, E.J.; FERREIRA, R.G.; SANTANA, M.F 2011. Inseticidas botânicos aplicados sobre Aphis gossypii e seu predador Cycloneda sanguínea em algodão colorido. Pesquisa Agropec. Brasileira. 46(11):1424-1431. 
3. BRITO, H.M.; GUEDES, M.; VARGAS, A.; GOMES, C.A. 2006. Toxicidade de Natuneem sobre Tetranychus urticae Koch (Acari: Tetranychidae) e ácaros predadores da família Phytoseiidae. Ciência e Agrotecnol. 30:75-78.

4. BUENO, V.H.P. 2005. Controle biológico de pulgões ou afídeos-praga em cultivos protegidos. Informe Agropecuário, Belo Horizonte. 26(225):9-17.

5. CAMPANHOLA, C.; BETTIOL, W. 2003. Panorama sobre o uso de agrotóxicos no Brasil. In: Campanhola, C.; Bettiol, W. (ed.). Métodos alternativos de controle fitossanitário. Jaguariúna. Embrapa Meio Ambiente. p.13-51.

6. CASTAGNOLI, M.; SIMONI, S.; GOGGIOLI, D. 2000. Attività biológica di sostanze vegetali nei confronti di Tetranychus urticae Koch (Acari: Tetranychidae) e del suo predatore Neoseiulus californicus (Mcgregor) (Acari: Phytoseiidae). Redia. 83:141-150.

7. CASTIGLIONI, E.; VENDRAMIM, J.D.; TAMAI, M.A. 2002. Evaluación del efecto tóxico de extractos acuosos y derivados de meliáceas sobre Tetranychus urticae (Koch) (Acari: Tetranychidae). Agrociência. 6:75-82.

8. D'ANDREA, P.A. 2001. Aspectos práticos e tendências no uso dos fertiprotetores. In: Hein, M. (org). Resumos do $1^{0}$ Encontro de Processos de Proteção de Plantas: controle ecológico de pragas e doenças. Agroecológica. Botucatu. p.97-101.

9. DIMETRY, N.Z.; AMER, S.A.A.; REDA, A.S. 1993. Biological activity of two neem seed kernel extracts against the two-spotted spider mite Tetranychus urticae Koch. J. Appl. Entomol. 116:308-312.

10. EASTERBROOK, M.A.; FITZGERALD, J.D.; SOLOMON, M.G. 2001. Biological control of strawberry tarsonemid mite Phytonemus pallidus and two-spotted spider mite Tetranychus urticae on strawberry in the UK using species of Neoseiulus (Amblyseius) (Acari: Phytoseiidae). Exp. Appl. Acarol. 25:25-36.

11. EL-GENGAIHI, S.; DIMETRY, N.Z.; AMER, S.A.A.; MOHAMED, S.M. 2000. Acaricidal activity of lipoidal matter of different plant extracts against the twospotted spider mite Tetranychus urticae Koch. Insect Sci. Appl. 20 (3):191-194.

12. FILGUEIRA, F.A.R. 2000. Novo manual de olericultura - agrotecnologia moderna na produção e comercialização de hortaliças. Viçosa: UFV. 402p.
13. FINNEY, D.J. 1971. Probit analysis. Cambridge: Cambridge University Press. 333p.

14. FRAGOSO, D.B.; GUEDES, R.N.C.; PICANÇO, M.C.; ZAMBOLIM, L. 2002. Insecticide use and organophosphate resistance in the coffee leaf miner Leucoptera coffeella (Lepidoptera: Lyonetiidae). Bull. Entomol. Res. 92:203-212.

15. GUERRA, M.S. 1985. Receituário caseiro: alternativa para o controle de pragas e doenças de plantas cultivadas e seus produtos. Brasília: EMATER. 166p.

16. GUIRADO, N. 2001. Extrato de plantas no controle da leprose dos citros. In: Hein, M. (org). PRIMER ENCONTRO DE PROCESSOS DE PROTEÇÃO DE PLANTAS: Controle ecológico de pragas e doenças. Agroecológica, Botucatu. p.147-159.

17. MANSOUR, F.A.; ASCHER, K.R.S. 1983. Effects of neem (Azadirachta indica) seed kernel extracts from different solvents on the carmine spider mite, Tetranychus cinnabarinus. Phytoparasitica. 11:177185.

18. MARTíNEZ, S.S. 2002. O nim Azadirachta indica: Natureza, Usos Múltiplos, Produção. Londrina, IAPARInstituto Agronômico do Paraná. 142p.

19. MICHELOTTO, M.D.; Silva, R.A.; Busoli, A.C. 2004. Tabelas de vida para Aphis gossypii Glover, 1877 (Hemiptera: Aphididae) em três espécies de plantas daninhas. Bol. San. Veg. Plagas. Madrid. 30(1):211-217.

20. MOMEN, F.M.; REDA, A.S.; AMER, S.A.A. 1997. Effect of Neem Azal-F on Tetranychus urticae and three predacious mites of the family Phytoseiidae. Acta Phytopath. Entomol. Hung. 32:355-362.

21. MOURÃO, S.A.; SILVA, J.C.T.; GUEDES, R.N.C.; VENZON, M.; JHAM, G.N.; OLIVEIRA, C.L.; ZANUNCIO, J.C. 2004. Seletividade de extratos de nim (Azadirachta indica A. Juss.) ao ácaro predador Iphiseiodes zuluagai (Denmark \& Muma) (Acari: Phytoseiidae). Neotrop. Entomol. 33:613-617.

22. OCETE, R.; LÓPEZ, M.A.; DANCSHÁZY, Z.; OCETE, M.E.; PÉREZ, M.A.; KAJATI, I.; RULL, G. 2003. IPM-sustainable tests on two apple tree pests, Eriosoma lanigerum Hausm (Homoptera: Aphididae) and Aphis pomi De Geer (Homoptera: Aphididae), carried out in La Rioja (Spain). Boletín de Sanidad Vegetal, Plagas. Ministerio de Agricultura, Pesca y Alimentación. p.35-45. 
23. PALLINI, A.; VENZON, M.; OLIVEIRA, H.G.; FADINI, M.A.M. 2004. Manejo Integrado de Pragas em cultivos protegidos. In: Aguilar, R.L.; Darezzo, R.J.; Rozane, D.E.; Aguilera, G.A.H. \& Silva, D.J.H. Cultivo em ambiente protegido. Viçosa, M.G. UFV. 1:207-224.

24. PENTEADO, S.R. 2000. Controle alternativo de pragas e doenças com as caldas bordalesa, sulfocálcica e Viçosa. Buena Mendes Gráfica e Editora, Campinas. 95p.

25. PICANÇO, M.C.; SOTO, A.; BACCI, L.; FIDELIS, E.G.; SILVA, G.A.; DE SENA, M.E. 2007. Controle biológico das principais pragas de hortaliças no Brasil. In: ZAMBOLIM, L. (Ed.). Manejo integrado de doenças e pragas hortaliças. Viçosa: UFV. p.505-537.

26. REMBOLD, H. 1989. Azadirachtina, their structure and mode of action. In: ARNASON, J.T.; PHILOGENE, B.J.R.; MORAND, P. (Eds.) Insecticides of plant origin. Washington: ACS.11. p.150-163.

27. SAS INSTITUTE. 1989. SAS User's Guide: Statistics, Version 9.0, 7th ed. SAS Institute, Cary, NC.

28. SOGLIA, M.C.M.; BUENO, B.H.P.; SAMPAIO, M.V. 2002. Desenvolvimento e sobrevivência de Aphis gossypii Glover (Hemiptera: Aphididae) em diferentes temperaturas e cultivares comercias de crisântemo. Neotrop. Entomol. 31(2):211-216.

29. SOTO, A. 2009. Control del ácaro Oligonychus ilicis con calda sulfocálcica. Revista Agronomía.. U. Caldas. 17(1):7-11.

30. SOTO, A.; VENZON, M.; OLIVEIRA, R.M.; OLIVEIRA, H.G.; PALLINI, A. 2010. Alternative control of Tetranychus evansi Baker \& Pritchard (Acari: Tetranychidae) on tomato plants grown in greenhouses. Neotrop. Entomol. 39(4):638-644.

31. SOTO, A.; OLIVEIRA, H.; PALLINI, A. 2011. Integración de control biológico y de productos alternativos con- tra Tetranychus urticae (Acari: Tetranychidae). Rev. U.D.C.A. Act. \& Div. Cient. 14 (1):23-29.

32. SPOLLEN, K.M.; ISMAN, M.B. 1996. Acute and sublethal effects of a neem insecticide on the commercial biological control agents Phytoseiulus persimilis and Amblyseius cucumeris (Acari: Phytoseiidae) and Aphidoletes aphidimyza (Diptera: Cecidomyiidae). J. Econ. Entomol. 89:1379-1386.

33. STARK, J.D.; VARGAS, R.I.; MESSING, R.H.; PURCELL, M. 1992. Effects of cyromazine and diazinon on three economically important Hawaiian tephritid fruit flies (Diptera: Tephritidae) and their endoparasitoids (Hymenoptera: Braconidae). J. Econ. Entom. 85:16871694.

34. STARK, J.D.; BANKS, J.E. 2003. Population - level effects of pesticides and other toxicants on arthropods. Ann. Rev. Entomol. 48:505- 519.

35. STARK, J.D.; RANGUS, T. 1994. Lethal and sublethal effects of the neem insecticide, Margosan-O, on pea aphid. J. Pest Sci. 41:155-160.

36. VÁSQUEZ, R.F. 2005. Evaluación de extractos vegetales en el control de insectos plaga a nivel de huerto familiar. Memoria de residencia. ITAO. No. 23 Oaxaca. México. 35p.

37. VENZON, M.; PALLINI, A.; FADINI, M.A.M.; OLIVEIRA, H.; MIRANDA, V.S.; DE ANDRADE, A.P.S. 2007. Controle alternativo de ácaros em hortaliças. In: ZAMBOLIM, L. (Ed.). Manejo integrado de doenças e pragas hortaliças. Viçosa: UFV. p.607-625.

38. WARDLOW, L.R.; LUDLAM, F.A.B. 1975. Biological studies and chemical control of brown scale (Parthenolecanium corni (Bouché)) on Red Currant. Plant Pathol. 24:213-216.

Recibido: Octubre 23 de 2012

Aceptado: Abril 22 de 2013 